\title{
Saturable Absorption of an X-Ray Free-Electron-Laser Heated Solid-Density Aluminum Plasma
}

\author{
D. S. Rackstraw, ${ }^{1, *}$ O. Ciricosta, ${ }^{1}$ S. M. Vinko, ${ }^{1}$ B. Barbrel, ${ }^{2}$ T. Burian, ${ }^{3}$ J. Chalupský, ${ }^{3}$ B. I. Cho, ${ }^{4,5}$ H.-K. Chung, ${ }^{6}$ \\ G. L. Dakovski, ${ }^{7}$ K. Engelhorn, ${ }^{2}$ V. Hájková, ${ }^{3}$ P. Heimann, ${ }^{7}$ M. Holmes, ${ }^{7}$ L. Juha, ${ }^{3}$ J. Krzywinski, ${ }^{7}$ R. W. Lee, ${ }^{8}$ S. Toleikis, ${ }^{9}$ \\ J. J. Turner, ${ }^{7}$ U. Zastrau, ${ }^{7,10}$ and J. S. Wark ${ }^{1}$ \\ ${ }^{1}$ Department of Physics, Clarendon Laboratory, University of Oxford, Parks Road, Oxford OX1 3PU, United Kingdom \\ ${ }^{2}$ Lawrence Berkeley National Laboratory, 1 Cyclotron Road, Berkeley, California 94720, USA \\ ${ }^{3}$ Institute of Physics ASCR, Na Slovance 2, 18221 Prague 8, Czech Republic \\ ${ }^{4}$ Department of Physics and Photon Science, Gwangju Institute of Science and Technology, Gwangju 500-712, Republic of Korea \\ ${ }^{5}$ Center for Relativistic Laser Science, Institute for Basic Science (IBS), Gwangju 500-712, Republic of Korea \\ ${ }^{6}$ Atomic and Molecular Data Unit, Nuclear Data Section, IAEA, P.O. Box 100, A-1400 Vienna, Austria \\ ${ }^{7}$ SLAC National Accelerator Laboratory, 2575 Sand Hill Road, Menlo Park, California 94025, USA \\ ${ }^{8}$ Department of Physics, University of California, Berkeley, California 94720, USA \\ ${ }^{9}$ Deutsches-Elektronensynchrotron DESY, Notkestrasse 85, 22603 Hamburg, Germany \\ ${ }^{10} I O Q$, Friedrich-Schiller-Universität Jena, Max-Wien-Platz 1, 07743 Jena, Germany
}

(Received 11 September 2014; published 9 January 2015)

\begin{abstract}
High-intensity $\mathrm{x}$-ray pulses from an $\mathrm{x}$-ray free-electron laser are used to heat and probe a solid-density aluminum sample. The photon-energy-dependent transmission of the heating beam is studied through the use of a photodiode. Saturable absorption is observed, with the resulting transmission differing significantly from the cold case, in good agreement with atomic-kinetics simulations.
\end{abstract}

The development of x-ray free-electron lasers (XFELs) [1-3], capable of producing intensities on target in excess of $10^{17} \mathrm{~W} \mathrm{~cm}^{-2}$, has opened up new regimes in $\mathrm{x}$-raymatter interactions. Considerable effort has been made to understand these intense $\mathrm{x}$-ray-matter interactions at various scales, from atoms [4,5], through molecules [6-8] and clusters [9-11], to solid samples [12,13]. X-rays from XFELs are intense enough that a single ion may interact with more than one photon during a single tens-offemtosecond pulse, with the possibility of creating ions that are fully stripped of their electrons [4], and the observation of nonlinear phenomena in the x-ray regime [14-16]. A key optical phenomenon that occurs at high intensities is that of saturable absorption, an observed effect that has recently been extended into the XUV region of the electromagnetic spectrum (close to the $L$ edge of aluminum at $92 \mathrm{eV}$ ) by the use of the FLASH FEL [17], and in the x-ray regime in the case of atoms, molecules, and clusters at the Linac Coherent Light Source (LCLS) [4,6,9]. In all of these works, saturable absorption is achieved by photoionizing the sample at a faster rate than that of the relevant recombination processes. Here we demonstrate a different wavelength-dependent saturable absorption mechanism where x-ray pulses heat a solid-density sample so efficiently that the $K$ edges of the ions present in the hot collisional plasma have energies exceeding the photon energy of the x-rays. Saturable absorption effects have very recently been reported in a solid sample driven by hard $\mathrm{x}$-rays at the SACLA FEL [18]. In contrast to that work, the experimental results and associated modeling presented here demonstrate that the increased transmission at these $\mathrm{x}$-ray intensities is due to the large blueshifts in the $K$ edges of the ions that are a direct result of both core-hole creation by the FEL and, upon rapid refilling, the consequent change in the number of $L$-shell electrons owing to Auger and radiative decay, as well as subsequent collisional ionization of outer-shell electrons.

We performed measurements of the transmission for a solid aluminum sample exposed to the x-ray pulses of the LCLS, at photon energies ranging from $1540 \mathrm{eV}$ (just below the $K$ edge of cold aluminum) to $1870 \mathrm{eV}$. As the system is isochorically ionized to high charge states [12], the absorption edges will be shifted from the neutral position, due to the binding of the electrons to the nucleus increasing with the charge of the ion. If the $\mathrm{x}$-ray photon energy is tuned to just above the $K$ edge of a particular ion, it is possible that by the end of the pulse there will be no ions in the focus of the beam that are of an ionization state where they can absorb a photon by $K$-shell photoionization. The unavailability of this dominant absorption process will increase the absorption length, leading to saturation of the absorption induced by the ionization of the sample.

The experiment was performed on the SXR beam line of the LCLS at the SLAC National Accelerator Laboratory [19]. The pulse length of the electron bunch was $100 \mathrm{fs}$, which gives an approximate upper bound on the length of the light pulse. The photon energy of the beam has a bandwidth of around $0.3 \%$, while the number of photons in the pulse fluctuates by about $15 \%$. The energy of each x-ray pulse was measured using a gas monitor detector (GMD) 
and found to be $\approx 2 \mathrm{~mJ}$ prior to transmission through the beam line focusing optics. The beam was focused using Kirkpatrick-Baez mirrors, and the spatial distribution of intensities within the focal spot was diagnosed by analyzing the craters ablatively formed in a suitable material that strongly absorbs the LCLS radiation [20,21]. The focal spot had an effective area of $7 \mu \mathrm{m}^{2}$, with a distribution well approximated by a super-Gaussian. The photodiode measuring the transmission signal was calibrated by removing the target, attenuating the x-ray beam over a range of values by the use of the gas attenuator, and comparing the diode signal with the energy measurements of the GMD. The beam line transmission of between $27 \%$ and $34 \%$ was taken from Ref. [22]. This procedure was performed for a number of different photon energies and with different filters in front of the photodiode. The diode signal was found to fluctuate on a shot-to-shot basis by about $5 \%$ relative to that deduced from the GMD reading.

In order to prevent saturation of the diode, attenuating filters were used in front of it. The energy content of a single LCLS pulse is such that the total attenuation needs to be of order $10^{4}$. The filters used were a $72.5 \mu \mathrm{m}$ carbon filter and two aluminum filters with thicknesses of 12.5 and $16.5 \mu \mathrm{m}$. These three filters were used in the photon energy ranges of $1540-1680,1670-1800$, and $1800-1870 \mathrm{eV}$, respectively. As the level of attenuation introduced by the filters is high, care must be taken in interpreting photonenergy-dependent structure in the experimental data, particularly when using the $\mathrm{Al}$ filters above $1670 \mathrm{eV}$. This is because the cold $\mathrm{Al}$ filters will themselves exhibit modulations in their absorption coefficient owing to EXAFS (x-ray absorption fine structure), which although small in terms of opacity (a few percent), owing to the high optical depth of the filter can result in modulations in the filter transmission of close to factors of 2 or so. In the analysis here, the mass absorption coefficient of Barkyoumb and Smith [23] was used for the Al filters.

The irradiated sample was a $1 \mu \mathrm{m}$ thick foil of aluminum oriented at $45^{\circ}$ to the incident FEL beam. The sample was supported by a nickel mesh that covered around $10 \%$ of the rear surface area of the target. As the target was rastered to a fresh region for each shot, it would be expected that around $10 \%$ of the results would not show the true transmission due to the beam hitting the mesh. The 3rd harmonic of the XFEL beam was rejected with high efficiency, as the mirrors transporting the beam to the end station have a sharp cutoff in transmission above photon energies of around $2.2 \mathrm{keV}$ [24].

The experimental values of the transmission of the irradiated $\mathrm{Al}$ foil were modeled using the collisionalradiative code SCFLY [25], which has been adapted for simulations of intense XFEL radiation interacting with matter [26]. A series of simulations are performed over a range of photon energies and over 5 orders of magnitude in $\mathrm{x}$-ray intensities, each with the same temporal profile.
A flattop temporal profile with a duration equal to that of the electron beam is used in the simulations: as transparency is achieved whenever the total number of photons in the pulse significantly exceeds the minimum number required to strip the sample up to the relevant charge state, the results are largely insensitive to variations in pulse duration and shape. In practice, as the XFEL traverses the target the opacity changes. This is accounted for by assuming the target is divided into four laminae, with the output intensity of one lamina (calculated from the input intensity and opacity) used as the input intensity to the subsequent lamina. The transmissions of individual intensities were weighted according to the experimentally diagnosed spatial distribution of intensity. The effect of self-radiation on the atomic populations is dealt with using an escape factor formalism: previous work has shown that more intricate treatment of the opacity is less necessary at photon energies above the $K$ edge [27].

Figure 1 shows the transmission of the FEL beam as a function of photon energy. Also shown in the figure are the cold $\mathrm{Al}$ transmission curve taken from the CXRO website $[28,29]$ alongside SCFLY simulations using pulses of duration $100 \mathrm{fs}$ and energy (on target) of $0.8 \mathrm{~mJ}$ - slightly greater than the nominal value from the beam line transmission - though the uncertainty of the beam line transmission measurement of Ref. [22] is quite large. The general trend of the experimental results is shown by a line to guide the eye, with those far-outlying data shots neglected - these are due to partial windows or small holes in the target (those above the trend) or shots where the FEL beam hit, or partially hit, the nickel mesh that supported the target.

Just above the cold $K$ edge one might expect there to be a sharp reduction in the transmission as the cold absorption length shortens by about an order of magnitude. However, in the experiment, the drop is not as severe as in the cold case, a feature which is in good agreement with the simulations. This high transmission occurs, as although

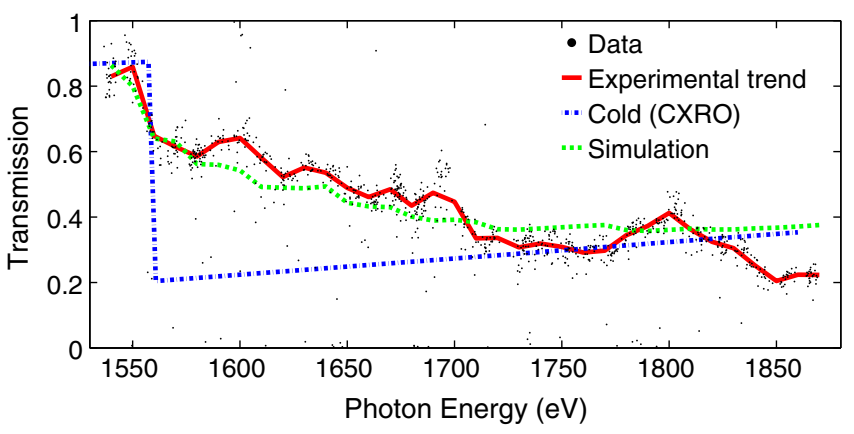

FIG. 1 (color online). The transmission of a $1 \mu \mathrm{m}$ thick aluminum sample oriented at $45^{\circ}$ to the FEL beam. The red line shows the experimental trend, neglecting outlying data points significantly far from the majority. The green line is from SCFLY simulations of the transmission. The blue line indicates the CXRO database values for the cold transmission. 
initially a larger number of photons will be absorbed early in the FEL pulse, later in the pulse the population of those ionic species that will absorb through $K$-shell photoionization has been diminished due to heating and ionization. This reduces the possible absorption mechanisms to primarily $L$-shell photoionization, which has a smaller cross section, giving a greater transmission.

In the cold case, after the edge, the transmission gradually increases as the photon energy increases. However, the experiment and simulations show the opposite trend, with the transmission decreasing as a function of increasing photon energy. For ions to become largely transparent to the FEL pulse, they need to be ionized to a degree such that their $K$ edge lies above the photon energy of the FEL. This will occur later and later in the FEL pulse as the photon energy is increased, due to the number of possible charge states that can be $K$-shell photoionized increasing with photon energy. This leads to the number of photons transmitted approaching the cold value at higher photon energies.

We note some fine structure in the transmission in both the experiment and the simulations. When the $K$ edge for the next higher charge state is reached, a new absorption pathway is opened, leading to greater absorption and a drop in transmission. The position of the drops agrees roughly with the $K$ edges shown in the work of Ciricosta et al. [30]. However, care must be taken in this interpretation, especially at photon energies above $1670 \mathrm{eV}$, because as previously noted some structure in the measured transmission may come about due to EXAFS in the cold filter. Although the EXAFS within the filter are taken into account in our analysis, any inaccuracies in their amplitude, as measured by Ref. [23], would result in some structure in the observed transmission.

In Fig. 2 we show the effective attenuation length of a 100 fs flattop pulse as a function of photon energy and fluence. The actual attenuation length will vary throughout the duration of the pulse, and so the effective attenuation length is calculated from the transmission assuming a

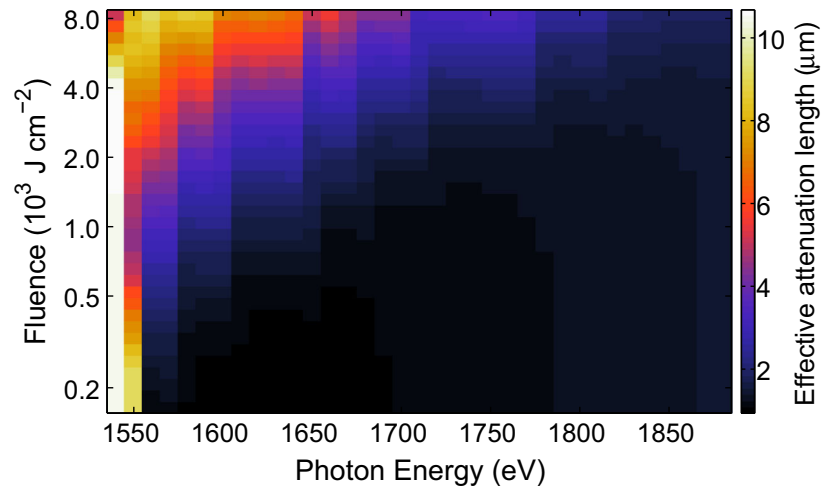

FIG. 2 (color online). Simulated effective attenuation length as a function of fluence at a range of central photon energies. The actual attenuation length will vary over the duration of the pulse. constant attenuation length throughout the pulse duration. The attenuation length is long below the cold $K$ edge for all intensities as expected. Above the cold $K$ edge, we note that the fluence at which the sample starts to transmit much more than the cold target increases as a function of increasing photon energy, owing to the need for more photons to be absorbed to achieve saturable absorption. Furthermore, as expected for the same reasons, for a given incident fluence the transmission drops as a function of photon energy.

Greater physical insight can be gained from studying the time-dependent evolution of the system in the simulations. Figure 3 shows the temporal evolution of the opacity of the sample when heated by a FEL tuned to $1670 \mathrm{eV}$. Contributions to the opacity from each of the charge states are also plotted, as is the evolution of the charge state distribution itself. The intensities of the FEL in these simulations are chosen to be $20 \%$ of the peak intensity
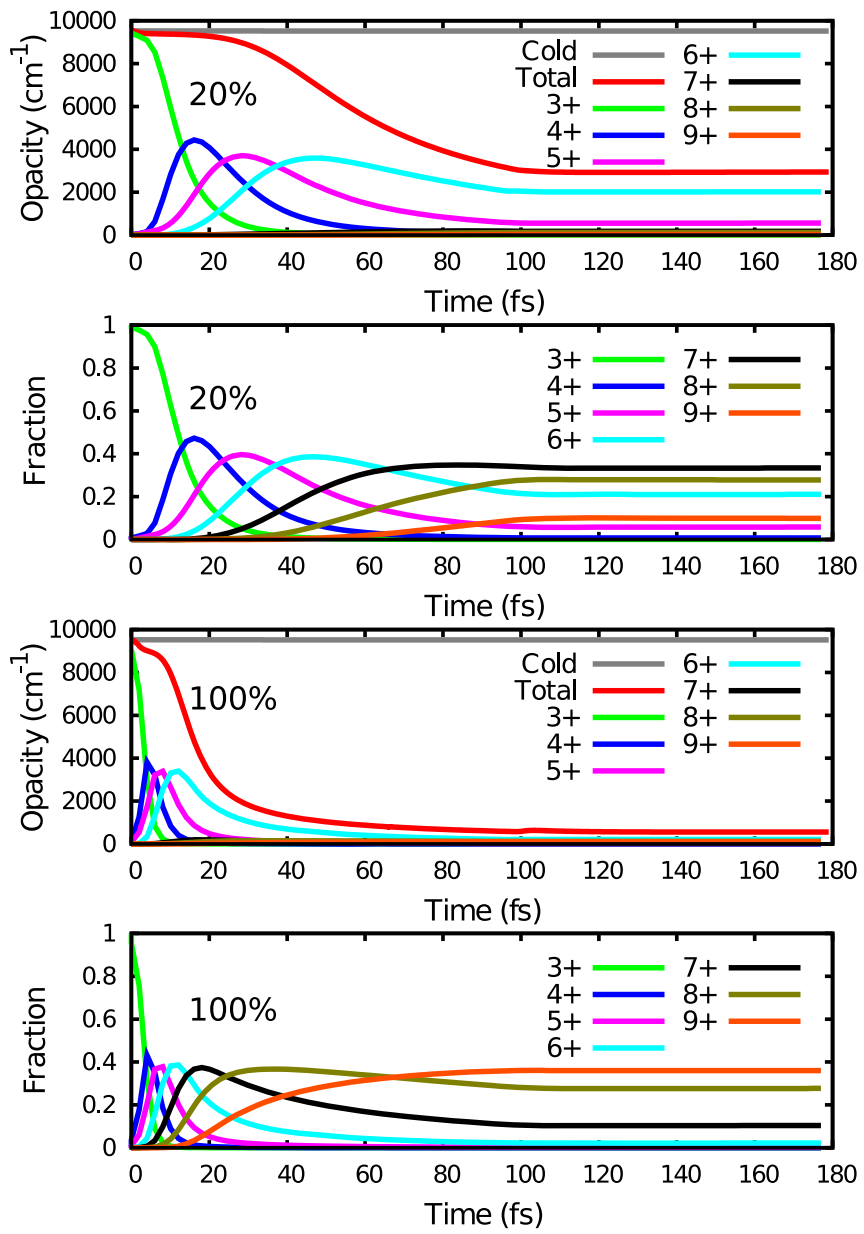

FIG. 3 (color online). Time-dependent opacities, with contributions from different charge states, and charge state distributions from simulations at a photon energy of $1670 \mathrm{eV}$ at $20 \%$ of peak intensity (top two panels) and the full intensity (bottom two panels). The FEL pulse is on for the first $100 \mathrm{fs}$ and constant in intensity. 
in the first set of plots and the full peak intensity in the second set. The transmission of the pulse at a given time is dependent on which charge states are present, with higher charge states being generated later in the pulse. It can be seen that, as expected, the opacity due to each charge state tracks the proportion of the sample in that charge state.

We note that initially the total opacity is constant and equal to the cold opacity value. In the simulation at $20 \%$ of the peak intensity, the opacity is approximately constant for the first $30 \mathrm{fs}$, after which it starts to drop, whereas in the higher intensity case the drop in opacity is very rapid indeed. This delay in the change in the opacity occurs because it takes time for the plasma to be ionized to a state where a significant number of the ions have charge states such that their $K$ edges lie above the FEL photon energy (which here is all ions above charge state 6+). Later in time, for both intensities, the opacity approaches a constant lower value. In the $20 \%$ intensity case, there is still a significant number of $6+$ ions present at the end of the pulse, and the opacity asymptotes to a constant value owing to the XFEL pulse switching off. In contrast, in the case of the simulation at the peak intensity, all of the $6+$ ions are burnt through within the first 50 to $60 \mathrm{fs}$, and a constant opacity (much lower than in the $20 \%$ intensity case) is achieved owing to the only absorption pathway being via the less probable $L$-shell ionization of the remaining (high) charge states.

This increase in transmission owing to a high $\mathrm{x}$-ray fluence is a demonstration of saturable absorption in the $\mathrm{x}$-ray region of the spectrum. Whereas saturable absorption has previously been observed in the XUV region with the FLASH FEL [17], it should be noted that the mechanisms in the two regimes are different. In the XUV case, saturation was achieved in a thin $\mathrm{Al}$ target when the intensity of the FEL was sufficient to directly compete with the Auger recombination. The intense XUV laser pulse photoionized an $L$-shell electron into the continuum, and the FEL photon energy was such that this caused the $L$ edge of the ion to increase in energy above the photon energy. However, rapid recombination of the ion was possible via Auger decay from the continuum (further heating the free electrons). As a result, saturation was achieved because the photoionization rate exceeded the Auger decay rate. In a similar way, saturable absorption was observed with very short pulses in atoms, molecules, and clusters at LCLS $[4,6,9]$. In contrast, in the experiment described here, saturation is achieved by heating the sample such that the final ionization states have $K$ edges lying higher in energy than the photon energy.

As saturation is achieved owing to ionization, it is important to understand how ionization proceeds within the target. While the FEL primarily promotes electrons from the $K$ shell into the continuum, subsequent rapid Auger decay of $L$-shell electrons then fills the $K$-shell holes (with a rate such that this occurs on a time scale short compared with the FEL pulse duration, in contrast to the XUV case). However, this Auger decay process promotes energetic electrons into the continuum, heating the freeelectron gas. This thermal bath of electrons can itself cause ionization via electron-ion collisions.

As an example, in Fig. 4 we plot the ionization rate of the $4+$ ion via various processes during the pulse. The excited state population, i.e., those ions with a $K$-shell hole, is generated through the direct photoionization by the FEL of the charge state below and thus it peaks prior to the groundstate population of this same charge state. Through the photoionization and subsequent Auger decay processes, the free electrons are heated as the $\mathrm{x}$-ray pulse is absorbed. This increases the collision rate such that after $10 \mathrm{fs}$ it exceeds the photoionization rate of the ground state and after $30 \mathrm{fs}$ exceeds the Auger decay rate of the excited state. Hence, at these high intensities it is through the collisional ionization that the final charge state distribution is achieved. Note, however, that for shorter laser pulse lengths the times above will be correspondingly reduced: the final charge stateand thus whether saturated absorption occurs-is ultimately determined by how much energy can be absorbed by the sample during the pulse, not by the pulse duration. This is in contrast with what is observed for atomic samples $[4,6,9]$, where the photoionization processes dominate the evolution of the charge states.

In summary, we have demonstrated saturable absorption in the x-ray regime and demonstrated that the absorption curve of aluminum heated by an XFEL has the opposite trend as a function of energy compared with that of the cold curve. The absorption profile of matter under intense irradiation by $\mathrm{x}$-rays has application in determining the coefficients in the multiwavelength anomalous diffraction method of determining phase in x-ray crystallography [31,32]. This saturable absorption occurs because the

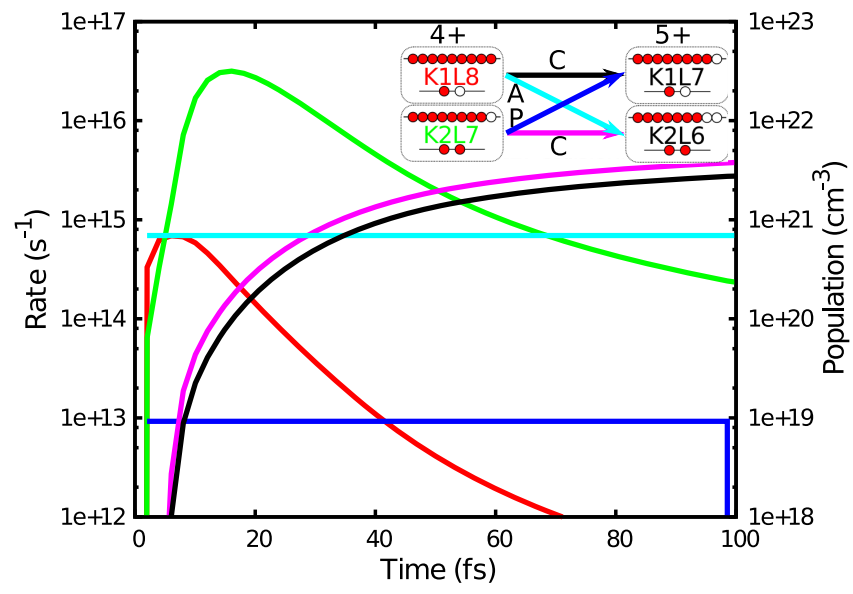

FIG. 4 (color online). The rates of ionization of the $4+$ charge state through photoionization (P), Auger (A), and collisional (C) processes. Also shown are the populations of the two superconfigurations of the $4+$ charge state. 
x-ray heated sample is collisionally ionized to such a degree that the $K$ edges of the ions present lie higher in energy than the photon energy.

Portions of this research were carried out on the SXR Instrument at the Linac Coherent Light Source (LCLS), a division of SLAC National Accelerator Laboratory and an Office of Science user facility operated by Stanford University for the U.S. Department of Energy. The SXR Instrument is funded by a consortium whose membership includes the LCLS, Stanford University through the Stanford Institute for Materials Energy Sciences (SIMES), Lawrence Berkeley National Laboratory (LBNL), the University of Hamburg through the BMBF priority program FSP 301, and the Center for Free Electron Laser Science (CFEL). D. S. R., O. C., S. M. V., and J. S. W. thank the U.K. EPSRC for funding (EP/F020449/1 and $\mathrm{EP} / \mathrm{H} 035877 / 1)$. B. I. C was supported by the NRF of Korea (No. 2013R1A1A1007084) and the TBP research project of GIST. T. B., V. H., J.Ch and L. J. appreciate a financial support from the Czech Science Foundation (Grant No. 14-29772S), Czech Ministry of Education (Grant No. LG13029) and Academy of Sciences of the Czech Republic (Grant No. M100101221). K. E. and P. H. acknowledge U.S. DOE Basic Energy Science Contract No. DE-AC03-76SF00098 and SSAA program Contract No. DE-FG52-06NA26212. U.Z. is grateful to the Volkswagen Foundation and the German Ministry for Education and Research via FSP-302.

*david.rackstraw@physics.ox.ac.uk

[1] W. Ackermann et al., Nat. Photonics 1, 336 (2007).

[2] P. Emma et al., Nat. Photonics 4, 641 (2010).

[3] T. Ishikawa et al., Nat. Photonics 6, 540 (2012).

[4] L. Young et al., Nature (London) 466, 56 (2010).

[5] B. Rudek et al., Nat. Photonics 6, 858 (2012).

[6] M. Hoener et al., Phys. Rev. Lett. 104, 253002 (2010).

[7] L. Fang, T. Osipov, B. Murphy, F. Tarantelli, E. Kukk, J. P. Cryan, M. Glownia, P. H. Bucksbaum, R. N. Coffee, M. Chen, C. Buth, and N. Berrah, Phys. Rev. Lett. 109, 263001 (2012).

[8] N. Berrah, L. Fang, T. Osipov, B. Murphy, C. Bostedt, and J. Bozek, J. Electron Spectrosc. Relat. Phenom. 196, 34 (2014).
[9] S. Schorb, D. Rupp, M. L. Swiggers, R. N. Coffee, M. Messerschmidt, G. Williams, J. Bozek, S.-I. Wada, O. Kornilov, T. Möller, and C. Bostedt, Phys. Rev. Lett. 108, 233401 (2012).

[10] H. Thomas et al., Phys. Rev. Lett. 108, 133401 (2012).

[11] T. Gorkhover et al., Phys. Rev. Lett. 108, 245005 (2012).

[12] S. M. Vinko et al., Nature (London) 482, 59 (2012).

[13] B. I. Cho et al., Phys. Rev. Lett. 109, 245003 (2012).

[14] G. Doumy et al., Phys. Rev. Lett. 106, 083002 (2011).

[15] C. Weninger, M. Purvis, D. Ryan, R. A. London, J. D. Bozek, C. Bostedt, A. Graf, G. Brown, J. J. Rocca, and N. Rohringer, Phys. Rev. Lett. 111, 233902 (2013).

[16] M. Beye, S. Schreck, F. Sorgenfrei, C. Trabant, N. Pontius, C. Schüß ler Langeheine, W. Wurth, and A. Föhlisch, Nature (London) 501, 191 (2013).

[17] B. Nagler et al., Nat. Phys. 5, 693 (2009).

[18] H. Yoneda, Y. Inubushi, M. Yabashi, T. Katayama, T. Ishikawa, H. Ohashi, H. Yumoto, K. Yamauchi, H. Mimura, and H. Kitamura, Nat. Commun. 5, 5080 (2014).

[19] W. F. Schlotter et al., Rev. Sci. Instrum. 83, 043107 (2012).

[20] J. Chalupsky, P. Bohacek, V. Hajkova, S. Hau-Riege, P. Heimann, L. Juha, J. Krzywinski, M. Messerschmidt, S. Moeller, B. Nagler, M. Rowen, W. Schlotter, M. Swiggers, and J. Turner, Nucl. Instrum. Methods Phys. Res., Sect. A 631, 130 (2011).

[21] J. Chalupský, T. Burian, V. Hájková, L. Juha, T. Polcar, J. Gaudin, M. Nagasono, R. Sobierajski, M. Yabashi, and J. Krzywinski, Opt. Express 21, 26363 (2013).

[22] K. Tiedtke et al., Opt. Express 22, 21214 (2014).

[23] J. H. Barkyoumb and D. Y. Smith, Phys. Rev. A 41, 4863 (1990).

[24] D. Ratner et al., Phys. Rev. ST Accel. Beams 14, 060701 (2011).

[25] H.-K. Chung, M. H. Chen, and R. W. Lee, High Energy Density Phys. 3, 57 (2007).

[26] O. Ciricosta, H.-K. Chung, R. W. Lee, and J. S. Wark, High Energy Density Phys. 7, 111 (2011).

[27] D. Rackstraw et al., High Energy Density Phys. 11, 59 (2014).

[28] B. Henke, E. Gullikson, and J. Davis, At. Data Nucl. Data Tables 54, 181 (1993).

[29] CXRO, http://www.cxro.lbl.gov, accessed: March 17, 2014.

[30] O. Ciricosta et al., Phys. Rev. Lett. 109, 065002 (2012).

[31] S.-K. Son, H. N. Chapman, and R. Santra, Phys. Rev. Lett. 107, 218102 (2011).

[32] S.-K. Son, H. N. Chapman, and R. Santra, J. Phys. B 46, 164015 (2013). 